[4] C. W. Barnes, "Roundoff noise and overflow in normal digital filters," IEEE Trans. Circuits Syst., vol. CAS-26, pp. 154-159, Mar. 1979.

[5] W. L. Mills, C. T. Mullis, and R. A.- Roberts, "Normal realizations of IIR digital filters," in 1979 IEEE Int. Conf. Acoustics, Speech, Signal Processing Rec., pp. 340-343, Apr. 1979.

[6] M. Arjmand and R. A. Roberts, "Reduced multiplier, low roundoff noise digital filters," in 1979 IEEE Int. Conf. Acoustics, Speech, Signal Processing Rec., pp. 344-346, Apr. 1979.

[7] T. H. Crystal and L. Erhman, "The design and application of digital filters with complex coefficients," IEEE Trans. Audio Electroacoust., vol. AU-16, pp. 315-320, Sept. 1968.

[8] L. B. Jackson, "Roundoff noise analysis for fixed-point digital filters realized in cascade or parallel form," IEEE Trans. Audio Electoacoust., vol. AU-18, pp. 107-122, June 1970.

[9] C. T. Mullis and R. A. Roberts, "Synthesis of minimum roundoff noise fixed point digital filters," IEEE Trans. Circuits Syst., vol. CAS-23, Sept. 1976.

[10] A. H. Gray, Jr. and J. D. Markel. "A normalized digital filter structure," IEEE Trans. Acoustics, Speech, Signal Processing, vol. ASSP-23, pp. 268-277, June 1975

[11] J. D. Markel and A. H. Gray, Jr., "Roundoff noise characteristics of a class of orthogonal polynomial structures," IEEE Trans. Acoustics, Speech, Signal Processing, vol. ASSP-23, pp. 473-485, Oct. 1975.

[12] J. D. Markel and A. H. Gray, Jr. "Fixed-point implementation algorithms for a class of orthogonal polynomial filter structures," IEEE Trans. Acoustics, Speech, Signal Processing, vol. ASSP-23, pp. 486-494, Oct. 1975 .

\section{Conditions for Finiteness of a Constructive Algorithm for Determining Stability}

\author{
JOHN N. TSITSIKI.IS
}

\begin{abstract}
In earlier papers [1], [2], a methodology for deciding on the stability of a system of nonlinear differential equations was proposed. This methodology reduced the problem to a test of boundedness of all finite products of a given finite set of matrices. This paper investigates further the issue of whether the algorithm will terminate in finitely many steps and obtains some new conditions, concentrating on the case where the system being tested is unstable.
\end{abstract}

\section{INTRODUCTION}

Brayton and Tong have proposed in [1] and [2] a constructive algorithm for determining the stability properties of a nonlinear time-invariant differential equation. They show that under certain conditions the problem may be reduced to the study of the "stability of a finite set of matrices", a concept to be defined below. They then proposed an algorithm that solves the latter problem and which is equivalent to constructing a Lyapunov function for the original differential equation. The range of potential applications of this algorithm is quite broad. It can be used, for example, in the study of switching systems, meaning linear systems such that the $A$ matrix may undergo sudden changes (either random or deterministic).

Given the importance of this algorithm, it is natural to ask under what conditions it will be constructive (i.e., it terminates

Manuscript received May 11, 1981; revised November 13, 1981, and December $21,1981$.

The author is with the Laboratory for Information and Decision Systems and the Department of Electrical Engineering and Computer Science, Massachussetts Institute of Technology, Cambridge, MA 02139. after a finite number of steps) and is, therefore, implementable in a computer. Some sufficient conditions are given in [1] and [2]. In this paper we obtain more conditions for the finiteness of the algorithm.

The termination of the algorithm is very closely linked to the properties of subsets of $\mathcal{C}^{n}$ that are left invariant under multiplication by a set of matrices. Most of the results in this paper involve characterizations of such invariant subsets.

Definition 1: A set of $n$ by $n$ complex matrices $Q$ is stable if for every neighborhood of the origin $U \subseteq \mathcal{C}^{n}$, there exists another neighborhood of the origin $V$ such that $M V \subseteq U, \forall M \in \mathbb{Q}^{*}$, where $\mathbb{Q}^{*}$ is the semigroup of matrices generated by $\mathbb{Q}$ (i.e., the set of all finite products of matrices in $Q$ ).

If $W \subseteq \mathcal{e}^{n}$, let $\mathcal{K}[W]$ be the convex hull of $W$.

Theorem 1: Given a finite set $\mathbb{Q}=\left\{M_{0}, M_{1}, \cdots, M_{m-1}\right\}$ of $m$ distinct $n$ by $n$ complex matrices. Let $W_{0} \subseteq e^{n}$ be a bounded, convex and symmetric polyhedral neighborhood of the origin. Define, for $k>0$

$$
W_{k}=\mathcal{K}\left[\bigcup_{i=0}^{\infty} M_{j}^{i} W_{k-l}\right]
$$

where $j \equiv(k-1)(\bmod m)$. Then $\mathcal{Q}$ is stable if and only if $W^{*} \equiv$ $\cup_{i=0}^{\infty} W_{i}$ is bounded.

Proof: See [1], p. 227.

Brayton and Tong's algorithm consists of constructing the set $W^{*}$. We should point out that if $W$ is a convex polyhedron it is uniquely determined by the finite set $E[W]$ of its extreme points. The algorithm is finite if and only if $W^{*}$ can be constructed in a finite number of steps. In particular, a necessary condition for finiteness is that $W^{*}$, as well as $W_{i}, i=1,2 \cdots$ are convex polyhedra. Concerning the first step of the algorithm (equation (1.1)), the following result is proved in [1]:

Theorem 2: Let $M$ be a matrix whose eigenvalues have magnitude $|\lambda(M)|<1$; then, for any bounded neighborhood of the origin $R$, there exists some $J$ such that $\cup_{i=0}^{\infty} M^{i} R=\cup_{i=0}^{J} M^{i} R$.

In order to complete the discussion, we must consider what happens if $M$ is stable but has eigenvalues with $\left|\lambda_{i}\right|=1$. In that case, it is necessary for finiteness that $M$ has no eigenvalue equal to $e^{i \theta}(\theta \in[0,2 \pi])$ with $\theta / 2 \pi$ irrational. However, this condition is not sufficient. (For an example, see [2, p. 1124].)

Concerning the second step of the algorithm, we have, when $Q$ is stable [2]:

Theorem 3: If a set $\mathcal{Q}$ of matrices is stable and there exists a positive $k$ such that $\left|\lambda_{i}(M)\right| \leqslant k<1$ for all $M \in \mathbb{Q}^{*}$, then the algorithm will terminate "stable" in a finite number of steps.

Similarly with the case of the first step, it is easy to prove the following necessary condition for finiteness: If there exists some $M \in \mathbb{Q}^{*}$, and an eigenvalue $\lambda$ of $M$ such that $\lambda=\exp [i \theta]$ and $\theta / 2 \pi$ is irrational, then the algorithm is not finite.

Now define a set $\Theta$ as follows: $\theta \in \Theta$ if and only if $0 \leqslant \theta \leqslant 2 \pi$ and $\exp [i \theta]$ is an eigenvalue of some $M \in Q^{*}$. Then, if the algorithm terminates, $\theta / 2 \pi$ has to be rational, $\forall \theta \in \Theta$. We also have the following result:

Proposition 1: If the algorithm is finite, then $\Theta$ is a finite set.

Proof: Suppose that $\theta \in \Theta$ and $\theta / 2 \pi$ is rational and has been written as an irreducible ratio $n / d$ of two integers. It is then easy to show that $W^{*}$ has at least $d$ extreme points. On the other hand, finiteness of the algorithm means, that $W^{*}$ has finitely many extreme points. Because there are only finitely many rational numbers in $[0,1]$ whose denominator is less or equal than a finite number, it follows that $\Theta$ is a finite set. 
As a conclusion, if the algorithm is constructive, then the set of eigenvalues of matrices in $\mathbb{Q}^{*}$ has to lie either in a subset of the complex plane whose closure does not intersect the unit circle (asymptotically stable systems) or intersects it at finitely many points corresponding to angles with rational $\theta / \pi$.

\section{Stopping Criteria when $\mathbb{Q}$ is not Stable}

If $\Theta$ is unstable, then $W^{*}$ is unbounded and it cannot be constructed in finitely many steps. In that case, we expect that there will be some way to detect unbounded growth and terminate the algorithm. Brayton and Tong have proved that if one of the two conditions presented below is met while the algorithm is implemented, then we may terminate the algorithm and conclude that $\Theta$ is unstable. However, this does not answer the question whether the algorithm is finite or not, because there is no guarantee that one of these stopping conditions will be eventually met. This issue is investigated in this section.

(IC1): First Instability Criterion [1, p. 228]

If there exists a $k$ such that $\partial W_{0} \cap \partial W_{k}=\varnothing$ then $W^{*}$ is unbounded. ( $\partial W$ is the boundary of $W$.)

(IC2): Second Instability Criterion [2, p. 1123]

Let $\mathscr{A}$ be a set of $m$ matrices. Suppose that $k \geqslant m$. Let

$$
\begin{aligned}
I_{k} & =\left(W_{k}\right)_{I} \cap\left(\cup_{j=0}^{k-1} E\left[W_{j}\right]\right) \\
N_{k} & =E\left[W_{k}\right]-\left(E\left[W_{k}\right] \cap E\left[W_{k-m}\right]\right)
\end{aligned}
$$

(where $(W)_{I}$ is the interior of $W$ and - is used to denote the difference of two sets). Suppose that there exists some $J$ such that for each "new point" $y \in N_{J}$, there exists an "interior point" $x \in I_{J}$ and some $M \in \mathbb{Q}^{*}$ such that $y=M x$. Then $\mathcal{Q}$ is unstable.

We assume that each $M \in \mathbb{Q}$ is stable, but $\mathbb{Q}$ is unstable, and we are looking for necessary and sufficient conditions, in terms of $\mathbb{Q}$ so that the algorithm eventually terminates when one of the above two stopping tests is used. For this, we need a sequence of preliminary results.

Lemma 1: If a set $V^{*} \subseteq e^{n}$ is convex, unbounded and symmetric, then there exists a $x \in V^{*}$, such that $x \neq 0$ and $r x \in V^{*}, \forall r \in R$.

Proof: Omitted.

Proposition 2: Suppose that $W^{*}$ (as defined in Theorem 1) is unbounded. Let $W_{c}$ be the closure of $W^{*}$. Then, either $W^{*}=e^{n}$ or $W_{c}$ is isomorphic to $V^{*} \oplus e^{n-m}$, for some closed and bounded $m$-dimensional convex sets $V^{*}$.

Proof: Let $U$ be the set of vectors $x \in \mathcal{C}^{n}$ such that $r x \in$ $W^{*}, \forall r \in R$. Let

$$
d=\frac{\sup \left\{|x|: x \in W_{0}\right\}}{\inf \left\{|x|: x \notin W_{0}\right\}}
$$

If $x \in W_{0}$, then

$$
\left|\frac{i x}{d}\right|=|x| \frac{\inf \left\{|x|: x \notin W_{0}\right\}}{\sup \left\{|x|: x \in W_{0}\right\}} \leqslant \inf \left\{|x|: x \notin W_{0}\right\} .
$$

Therefore, $i x / d \in W_{0}$. Now suppose that $z \in U$ and $r \in R$. Then, $r d z \in U$ and in particular $r d z \subset W_{c}$. Therefore, there exists a sequence $\left\{w_{n}\right\}$ of points of $W_{0}$ and a sequence $\left\{M_{n}\right\}$ of matrices in $\mathbb{Q}^{*}$ such that $M_{n} x_{n} \rightarrow r d z$. Then, $M_{n}\left(i x_{n} / d\right) \rightarrow r i z$. Since $i x_{n} / d \in$ $W_{0}$ and since $W_{c}$ is closed, we have $i r z \in W_{c}$. This implies that $i z \in U$ whenever $z \in U$ and consequently that $c z \in U, \forall z \in U$, $\forall c \in \mathcal{C}$

Now let $z_{1} \in U, z_{2} \in U$. Then, $2 c z_{1}, 2 c z_{2} \in W^{*}, \forall c \in \mathcal{C}$. By con-

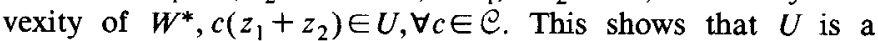
subspace of $e^{n}$ and is therefore isomorphic to $e^{n-m}$ for some $m$. If $m-n$, than $u / *-\rho n$ and wra nus dnm

Now, suppose that $m \neq 0$. Let $Q$ be the orthogonal comple- ment of $U$ and let $V^{*} \equiv Q \cap W_{c}$. Then, $V^{*}$ is closed and is equal to the intersection of two convex and symmetric sets. Therefore, it is also convex and symmetric. By construction of $V^{*}, V^{*} \cap U=\{0\}$ and there is no $x \in V^{*}$ such that $x \neq 0$ and $r x \in V^{*}, \forall r \in R$. Then, by Lemma $3, V^{*}$ is bounded.

We now have to show that $U$ and $V^{*}$, as constructed above, satisfy $V^{*} \oplus U=W_{c}$. Let $x_{1} \in V^{*}$ and $x_{2} \in U$. Then, $n x_{2} \in U, \forall n$ and $(1-1 / n) x_{1}+(1 / n)\left(n x_{2}\right) \in W_{c}$ by convexity. The above sequence of points converges to $x_{1}+x_{2}$ as $n \rightarrow \infty$. Since $W_{c}$ is closed, $x_{1}+x_{2} \in W_{c}$. Therefore, $W_{c} \supset V^{*} \oplus U$.

Consider now some $x \in W_{c}$. Let $x=x_{1}+x_{2}$ where $x_{2} \in U$ and $x_{1}$ belongs to the orthogonal complement of $U$. Then, $-n x_{2} \in U$, $\forall n$ and by convexity of $W_{c}$ we have $(1-1 / n)\left(x_{1}+x_{2}\right)+$ $(1 / n)\left(-n x_{2}\right) \in W_{c}$. We let $n \rightarrow \infty$ and since $W_{c}$ is closed, we conclude that $x_{1} \in W_{c}$. Therefore, $x_{1} \in V^{*}$ and $W_{c} \subset V^{*} \oplus U$.

Proposition 3: If $W_{c}$ is isomorphic to $V^{*} \oplus \mathfrak{e}^{n-m}$, with $m \neq 0$, then there exists a basis for $\mathcal{C}^{n}$ such that every $M \in \mathbb{Q}$ can be written as

$$
M=\left[\begin{array}{cc}
M_{11} & 0 \\
M_{21} & M_{22}
\end{array}\right]
$$

and the set $\left\{P_{1} M: M \in \mathbb{Q}\right\}$ is a stable set of matrices, where $P_{1}$ is the projection onto the subspace generated by the first $m$ coordinates.

Proof: Since $W_{c}$ is isomorphic to $V^{*} \oplus \mathfrak{C}^{n-m}$, there exists a basis such that $V^{*}$ belongs to the subspace generated by the first $m$ basis vectors. Using this basis, partition the matrix $M$ as follows:

$$
M=\left[\begin{array}{ll}
M_{11} & M_{12} \\
M_{21} & M_{22}
\end{array}\right] .
$$

Let $P_{2}$ be the projection on the subspace generated by the last $n-m$ basis vectors. Then, $P_{2} x \in W_{c}, \forall x \in \mathcal{e}^{n}$ which implies that $M P_{2} x \in W_{c}, \forall x \in \mathcal{C}^{n}$ and, therefore, $P_{1} M P_{2} x \in V^{*}, \forall x \in \mathcal{C}^{n}$. But this is another way of saying that

$$
\left[\begin{array}{cc}
0 & M_{12} \\
0 & 0
\end{array}\right] x \in V^{*}, \quad \forall x \in e^{n} .
$$

Since $V^{*}$ is bounded, we must have $M_{12}=0$. The stability of the set of matrices $P_{1} M$ follows from the fact that this set leaves the bounded set $V^{*}$ invariant.

Proposition 3 shows that $W^{*}=e^{n}$, except for some rather special cases that correspond to systems that may go unstable without having all modes go to infinity. It turns out that this is the class of systems for which the termination of the algorithm is uncertain.

Proposition 4: If $W^{*}=e^{n}$, then the stopping criterion $\mathrm{ICl}$ is met after finitely many steps.

Proof: If $\mathrm{ICl}$ is never met, there exists, for any $k$, a point $x_{k} \in \partial W_{0} \cap \partial W_{k}$. Since $W_{k}$ is an increasing sequence of sets, it follows that $x_{k} \in \partial W_{i}, \forall i<k$. Therefore, the sequence of compact sets $\partial W_{k}$ is decreasing and has the finite intersection property. This implies that $\cap_{k=0}^{\infty} \partial W_{k} \neq \varnothing$ which contradicts the fact $W^{*}-$ $e^{n}$.

Proposition 5: If $W^{*}=e^{n}$, then IC2 is met after finitely many steps.

Proof: As pointed out in [2, p. 1123], if $\mathscr{Q}$ is unstable, if IC2 is used and if the algorithm does not terminate, then there exists some $x_{j} \in W_{j}$ which does not become interior to any later constructed $W_{i}, i>j$. However, this contradicts the assumption $\cup_{i=j}^{\infty} W_{i}=\mathrm{e}^{n}$.

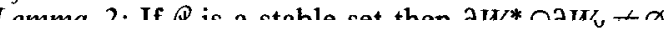

Proof: Let $k W \equiv\{k w: w \in W\}$. If $\partial W^{*} \cap \partial W_{0}=\varnothing$, then $\exists k$ 
$>1: k W_{0} \subset W^{*}$. From the definition of $W^{*}, W^{*}=\cup_{M \in Q^{*}} M W_{0}$ and since $\mathbb{Q}$ leaves $W^{*}$ invariant $W^{*}=\cup_{M \in \mathbb{Q}^{*}} M W^{*}$. Now, since $k W_{0} \subset W^{*}, W^{*} \supset \cup_{M \in q^{*}} M W_{0}=k\left(\cup_{M \in \mathbb{Q}^{*}} M W_{0}\right)$ and $W^{*} \supset k W^{*}$ which is a contradiction because we had assumed that $k>1$.

Proposition 6: If $W^{*}$ is isomorphic to $V^{*} \oplus \mathfrak{C}^{n-m}, m \neq 0$ then $\mathrm{ICl}$ is never met.

Proof: Assume that the change of basis suggested by Proposition 3 has been made and let $V_{k}$ be the projection of $W_{k}$ on the subspace generated by the first $m$ coordinates $\left(V_{k}=P_{1} W_{k}\right)$. Then, $\cup_{i=0}^{\infty} V_{k}=V^{*}$ and it is easy to check that the sequence $\left\{V_{k}\right\}$ is generated by the constructive algorithm when the set $\left\{P_{1} M: M\right.$ $\in \mathbb{Q}\}$ is used (and viewed as a set of matrices that operate on the $m$-dimensional subspace) and the initial convex neighborhood of the origin is taken to be $V_{0}$. Since $\left\{P_{1} M: M \in \mathbb{Q}\right\}$ is a stable set (by Proposition 3), we have $\partial V_{0} \cap \partial V^{*} \neq \varnothing$ (by Lemma 2).

Let $x \in \partial V_{0} \cap \partial V^{*}$ and let $z \in W_{0}$ be a point whose projection is $x$. Then, it is easy to see that $z \in \partial W_{0}$ and $z \in \partial W^{*}$. Therefore $z$ is interior to no $W_{k}$ and $\mathrm{ICl}$ is never met.

Propositions 4 and 6 contain the necessary and sufficient conditions that the algorithm terminates in a finite number of steps for an unstable system, when the stopping criterion is IC1. We have also shown that $W^{*}=e^{n}$ is a sufficient condition for termination when IC2 is used. However, it is unclear what the necessary conditions for termination are, when IC2 is used.

\section{ACKNOWLEDGMENT}

The author would like to thank Prof. John Wyatt for suggesting and encouraging this work.

\section{REFERENCES}

[1] R. K. Brayton and C. H. Tong, "Stability of dynamical systems; a constructive approach," IEEE Trans. Circuits Syst., pp. 224-234, Apr. 1979.

[2] - - "Constructive stability and asymptotic stability of dynamical systems," IEEE Trans. Circuits Syst., pp. 1121-1130, Nov. 1980.

\section{Synthesis of Generalized Interdigital Directional Couplers}

\section{RAMAMURTY NEDUNURI}

A bstract - In this paper a new kind of directional coupler is introduced and a method of synthesizing such couplers is described. The directional coupler described in this paper can be constructed in the generalized interdigital form.

\section{INTRODUCTION}

In [1] Rhodes has developed the theory of generalized interdigital two-port networks, and in [2] he has described a method of synthesizing generalized interdigital linear-phase filters. In this paper we show that a generalized interdigital four-port network can be used as a directional coupler. Also, we describe a method of synthesizing such directional couplers.

Manuscript received June 9, 1981 ; revised November 4, 1981.

The author is at 6, Dunkirk Rise, Rochdale, Lancashire OL12 $6 \mathrm{UH}$, England.

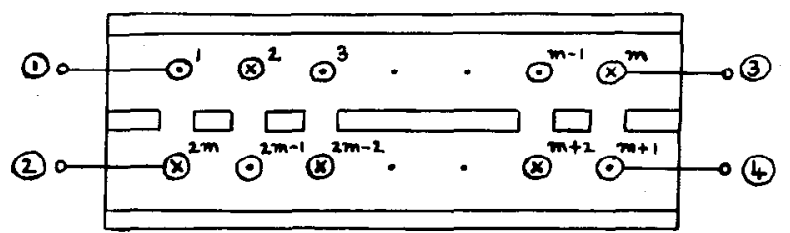

(a)

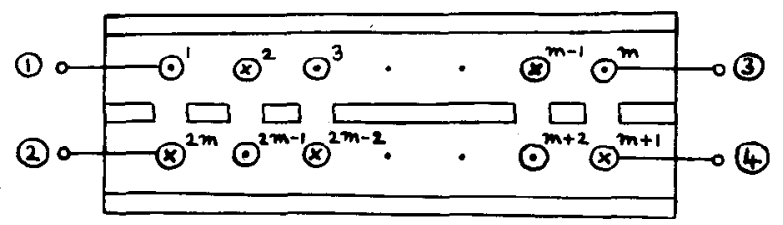

(b)

Fig. 1. Generalized interdigital four-port network. (a) $m$ even. (b) $m$ odd. (Conductor $\bullet$ is short-circuited to ground at the far end and conductor $X$ is short-circuited to ground at the near end.)

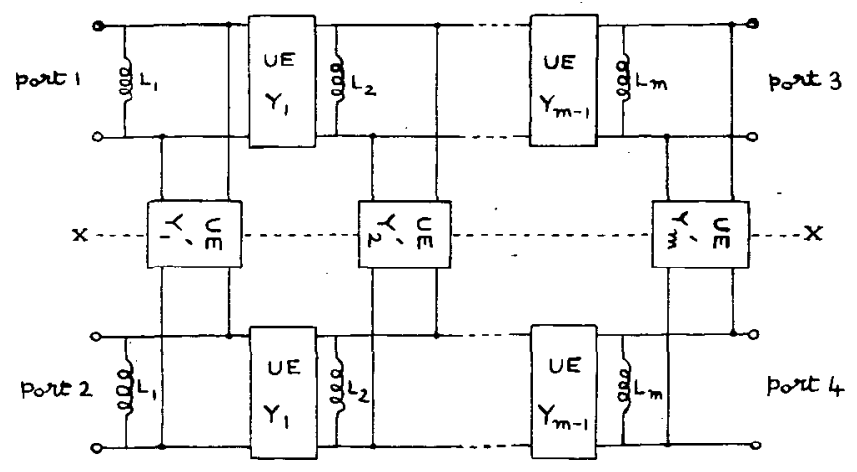

Fig. 2. $\lambda$-plane equivalent circuit of the generalized interdigital four-port network.

\section{Synthesis Procedure}

The generalized interdigital four-port network is shown in Fig. 1. Each half of this skew-symmetric structure is a conventional interdigital network without nonadjacent coupling. Coupling between the two sets of conductors is through slots in a coupling plate located between the two portions. The $\lambda$-plane equivalent circuit of the four-port network is shown in Fig. 2 [2]. ${ }^{1}$ The analysis of the symmetrical four-port network of Fig. 2 may be based on the concept of even- and odd-mode networks [3]. To construct these two-port networks we first bisect the symmetrical four-port network into halves along the line of symmetry $X X$. The even-mode network is the two-port network obtained when the cut terminals of one of the halves are open-circuited, and the odd-mode network is the two-port network obtained when the cut terminals are short-circuited. Using the new complexfrequency variable $z=\rho+j \mu=\tanh (a p / 2),{ }^{2}$ we can represent the even- and odd-mode networks in the forms shown in Fig. 3. Let

$$
S_{e}(z)=\left[\begin{array}{ll}
s_{11 e} & s_{12 e} \\
s_{12 e} & s_{22 e}
\end{array}\right]
$$

\footnotetext{
${ }^{1}$ Here $\lambda$ is the distributed complex-frequency variable defined by $\lambda=\gamma+j \Omega$ $=\tanh a p$, where $p=\sigma+j \omega$ is the true complex-frequency variable and $a$ is the delay time of the transmission line.

${ }^{2}$ For $p=j \omega$ we have $\lambda=j \Omega=j \tan a \omega$ and $z=j \mu=j \tan (a \omega / 2)$. Therefore, for real frequencies we have $\Omega=\tan a \omega$ and $\mu=\tan (a \omega / 2)$.
} 